\title{
牦牛分子遗传多样性研究进展
}

马志杰 ${ }^{1}$, 钟金城 ${ }^{2}$, 韩建林 ${ }^{3}$, 徐惊涛 ${ }^{1}$, 刘仲娜 ${ }^{2}$, 白文林 ${ }^{4}$

1. 青海大学畜牧兽医科学院, 青海省放牧家畜营养与生态国家重点实验室培育基地, 青海高原牦牛研究中心, 西宁 810016;

2. 西南民族大学生命科学与技术学院, 成都 610041 ;

3. 中国农业科学研究院北京畜牧兽医研究所, 中国农业科学院一国际家畜研究所畜禽与牧草遗传资源联合实验室, 北京 100193;

4. 沈阳农业大学畜牧兽医学院, 沈阳 110866

摘要: 遗传多样性研究可有效地揭示牦牛的遗传变异, 是牦牛群体遗传学研究的主要内容之一。自 20 世纪 70 年代以来, 人们已对牦牛的体形外貌特征、染色体核型(带型)、生理生化特性和 DNA 序列变异等进行了较为深 入地研究。随着分子遗传学和 DNA 测序技术的迅猛发展, 近年来的研究主要集中在牦牛的分子遗传多样性。 文章对近 15 年来牦牛 mtDNA 和核基因组分子标记及侯选基因多样性的研究现状进行了综述, 对前景进行展望, 以期为牦牛群体基因组学等研究提供依据。

关键词：牦牛; 遗传多样性; 核基因组; 线粒体 DNA; 分子标记; Y 染色体

\section{Research progress on molecular genetic diversity of the yak (Bos grunniens)}

\author{
MA Zhi-Jie ${ }^{1}$, ZHONG Jin-Cheng ${ }^{2}$, HAN Jian-Lin ${ }^{3}$, XU Jing-Tao ${ }^{1}$, LIU Zhong-Na ${ }^{2}$, BAI \\ Wen-Lin ${ }^{4}$ \\ 1. Academy of Animal Science and Veterinary Medicine of Qinghai University, National Key Lab Cultivating Base of Plateau \\ Grazing Animal Nutrition and Ecology in Qinghai Province, Qinghai Plateau Yak Research Center, Xining 810016, China; \\ 2. College of Life Science \& Technology, Southwest University for Nationalities, Chengdu 610041, China; \\ 3. Chinese Academy of Agricultural Sciences-International Livestock Research Institute Joint Laboratory on Livestock and \\ Forage Genetic Resources, Institute of Animal Sciences, Chinese Academy of Agricultural Sciences, Beijing 100193, \\ China; \\ 4. College of Animal Science and Veterinary Medicine, Shenyang Agricultural University, Shenyang 110866, China
}

\begin{abstract}
Studies on genetic diversity, as the core of population genetics, reveal genetic variations of the yak (Bos grunniens). Since the 1970 s, the morphological, chromosomal, physiological, and biochemical characteristics, as well as DNA sequence polymorphisms, in yak have been extensively investigated. Following the rapid development of molecular genetics and DNA sequencing technology, the molecular genetic diversity of yak has become a focus in recent studies. In this paper, the research progress on the molecular genetic diversity of yak was reviewed based on the information and knowledge on mtDNA sequences and nucleus molecular markers, as well as candidate genes, obtained over the last 15 years.
\end{abstract}

收稿日期: 2012-10-15; 修回日期: 2012-11-29

基金项目: 国家重点基础研究计划(973 计划前期研究专项)项目(编号：2012CB722906), 公益性行业(农业)科研专项(编号：201203008)、国家 国际科技合作专项(编号：2013DFA31420)和青海大学中青年基金团队项目资助

作者简介: 马志杰, 硕士, 副研究员, 研究方向 : 高原动物遗传育种与繁殖。E-mail: maziwise2003@yahoo.com.cn 
The future perspectives of relevant research topics were discussed to shed more light on depth understanding of the population genomics of the yak.

Keywords: yak; genetic diversity; nuclear genome; mitochondrial DNA; molecular marker; Y chromosome

牦牛(Bos grunniens) 是分布在海拔 2000 5500 m 以青藏高原为中心, 及其毗邻的高山、亚高山地区 的珍奇稀有牛种。目前, 全世界拥有约 1400 万头家 牦牛和 15000 头野牦牛, 其中中国拥有世界上最多 的牦牛数量和品种 (群体 $)^{[1,2]}$, 这些宝贵的遗传资源 是牦牛遗传多样性的物质基础。牦牛遗传多样性的 研究可为探究牦牛遗传资源的整体状况、起源驯化 模式、历史发展动态、品种类型划分以及合理保护 和开发利用等奠定理论基础。自 20 世纪 70 年代以 来，人们已从体态外貌特征、染色体核型(带型)、生 理生化特征和DNA序列变异等不同层次和水平对牦 牛的遗传多样性进行了较为深入的研究。特别是近 15 年来, 随着分子遗传学和测序技术的迅猛发展, 从分子水平上探究牦牛的遗传多样性已成为揭示牦 牛群体遗传变异的主要途径。自多位学者提出牦牛 在多个层次上具有丰富的遗传多样性后 ${ }^{[3 \sim 6]}$, 当前 已有大量有关牦牛分子遗传多样性的文献资料涌 现。尽管欧江涛等 ${ }^{[7]}$ 和Groeneveld等 ${ }^{[8]}$ 先后对牦牛的 分子遗传多样性研究进展进行了阐述, 但仍然未能 对最新进展进行系统地论述和探讨。鉴于此，本文 对近 15 年有关牦牛mtDNA和核基因组分子标记及 侯选基因遗传多样性的研究现状进行了综述, 并对 其前景作了展望, 以期为系统地开展牦牛群体基因 组学等研究提供依据。

\section{1 核基因组分子标记和侯选基因的遗传多 样性}

\section{1 核基因组分子标记}

\subsection{1常染色体分子标记}

电泳和测序技术的发展为从分子水平上准确的 评估牦牛的群体遗传多样性提供了契机。自 20 世纪 90 年代后期, 随机扩增片段长度多态性(Random amplified polymorphic DNA, RAPD)、扩增片段长度 多态性 (Amplified fragment length polymorphism,
AFLP)、相关序列扩增多态性(Sequence-related amplified polymorphism, SRAP)和微卫星(Simple sequence repeat, SSR)标记相继被运用于牦牛分子遗 传多样性的评估。

在使用RAPD、AFLP、SRAP标记进行牦牛品种 (群体)的分子遗传多样性研究中, 魏亚萍等 ${ }^{[9]}$ 、伍红 等 ${ }^{[10]}$ 和江明锋等 ${ }^{[11]}$ 对一些RAPD和AFLP标记在牦 牛及犏牛中的研究可行性进行了初步探索。钟金城 等 ${ }^{[12]}$ 和肖玉萍等 ${ }^{[13,14]}$ 采用RAPD和AFLP标记对九 龙、麦洼、大通和天祝白牦牛进行了分析，表明九 龙牦牛遗传多样性最丰富, 天祝白牦牛遗传多样性 最低, 4 个牦牛品种聚为两大类: 九龙牦牛为一类, 其它 3 个牦牛品种聚为一类。此后，钟金城等 ${ }^{[15]}$ 和 柴志欣等 ${ }^{[16]}$ 用RAPD引物对西藏 11 个牦牛群体进行 了研究, 表明西藏牦牛类群内具有较多的遗传变异, 西藏东部牦牛类群相比西部类群遗传多样性较高, 推测西藏东部可能是牦牛的起源地之一; 西藏牦牛 类群可分为两大类: 帕里牦牛为一类, 其余类群为 另一类。此外，赵上娟等 ${ }^{[17]}$ 首次将 4 对SRAP引物用 于西藏 11 个牦牛类群和四川麦洼牦牛的遗传多样 性和分类关系分析，表明 12 个类群的遗传多样性较 低; 聚类关系显示嘉黎、帕里、类乌齐、桑桑、康 布、巴青、丁青、斯布和麦洼牦牛聚为一大类, 然 后依次与桑日、工布江达和江达牦牛相聚在一起; 同时SRAP标记显示部分群体的亲缘关系与其地理 分布不一致。而在国外研究中, Ramesha等 ${ }^{[18]}$ 使用 RAPD标记对印度的 3 个牦牛群体进行了遗传分化 分析, 结果显示各牦牛群体间无显著差异。可以看 出, 以上 RAPD(Shannon 遗传多样性指数： 0.186 0.405)、AFLP (Shannon遗传多样性指数： 0.130 0.268)标记研究中其遗传多样性指数揭示中 国的麦洼、九龙、大通、天祝白牦牛以及西藏各牦 牛群体均具有丰富的遗传多样性, 且比印度牦牛群 体的多样性更为丰富, 但SRAP(Shannon遗传多样性 指数：0.014 0.146)标记揭示西藏各牦牛群体遗传多 
样性贫乏, 体现出不同标记在评估牦牛遗传多样性 状况时的偏差。然而, 以上各标记均提示上述各牦 牛品种(群体)内遗传变异比品种(群体)间更为丰富, 品种(群体)间存在一定程度的遗传分化, 其亲缘关 系与地理分布不一致。同时, RAPD研究支持将中国 牦牛划分为两个类型即青藏高原型和横断高山型的 观点, 并推测西藏东部可能是牦牛的起源驯化地之

相比以上各种分子标记, SSR标记具有结果的 重复性和可比性较高的特点, 是目前应用最广泛的 分子标记之一。近年来, SSR标记被广泛用于开展牦 牛品种(群体)的遗传多样性及相关研究。Ritz等 ${ }^{[19]}$ 最 早利用 20 个牛SSR标记对牛族 17 个代表性群体进 行系统发育分析, 结果显示普通牛和瘤牛先聚为一 类, 再依次与牦牛、大额牛、美洲野牛和水牛相聚。 王敏强 ${ }^{[20]}$ 和胡江 ${ }^{[21]}$ 分别对野牦牛以及甘南、大通和 天祝白牦牛的 9 个SSR位点进行分析, 表明家、野牦 牛均具有丰富的遗传多样性。Dorji等 ${ }^{[22]}$ 对 3 个不丹 牦牛群体进行SSR标记分析, 显示西部和中部牦牛 群体关系较近; 西部、中部牦牛群体和东部牦牛群 体至少在 4000 年前被隔离; 不丹牦牛群体可整体上 划分为两个类型 : 西部、中部牦牛群体和东部牛牛 群体。Wang等 ${ }^{[23]}$ 使用 12 个SSR标记对大通、甘南牦 牛进行分析表明, 两个群体间具有一定的遗传差 异。Qi ${ }^{[24]}$ 利用SSR标记结合系统发育地理学方法将 帕里牦牛归属于喜马拉雅山类群。同时, $\mathrm{Li}$ 等 ${ }^{[25]}$ 成功 构建了牦牛基因组微卫星富集文库, 并利用 20 个 SSR标记对 6 个牦牛品种(群体)进行分析, 提示品种 (群体)具有丰富的遗传多样性, 聚类结果表明麦洼 牦牛单独为一类, 而九龙、西藏拉萨、天祝、大通 和半血野牦牛聚为一类。而 Nguyen等 ${ }^{[26]}$ 以 10 头牦 牛为研究对象, 检测了 131 个普通牛SSR标记引物, 发现 117 个标记在牦牛中具有多态; 随后 26 个多态 SSR标记用于 51 头瑞士牦牛的多样性检测, 其群体 杂合度在 0.348 0.823 之间; 一套 13 个SSR标记组成 的引物用于父母和两头后代牦牛的亲子鉴定, 其准 确率为 $99.5 \%$ 。此外, Qi等 ${ }^{[27]}$ 使用 15 个SSR标记对 5 个蒙古牦牛群体、1 个俄罗斯牦牛群体和 1 个中国 牦牛群体(嘉黎牦牛)进行了遗传分化分析, 结果表 明被检测牦牛群体未发现瓶颈效应, 蒙古、俄罗斯 牦牛群体内具有丰富的遗传多样性, 但群体间存在
显著的遗传分化, 提示应该将这些牦牛群体在品种 保护和育种中看作独立的遗传“单元”。钟金城等 [12,28] 对 4 个牦牛品种进行SSR标记遗传多样性及其 分类研究，表明牦牛品种间和品种内均具有丰富的 遗传多样性; 聚类分析分为两大类, 即九龙牦牛单 独成一大类, 其它麦洼、大通、天祝白牦牛聚为一 类。毛永江等 ${ }^{[29]}$ 对青海高原牦牛进行SSR多态性、 中性检验及连锁不平衡检测, 表明各SSR位点全部 为多态, 群体内遗传变异水平较低, 各位点在群体 水平上均处于连锁平衡状态。与此同时, Zhang等 ${ }^{[30]}$ 、 廖信军等 ${ }^{[31]}$ 利用 16 个SSR标记对中国 9 个牦牛群体 (包括九龙、麦洼、西藏高山、斯布、帕里、青海高 原、中甸、新疆和天祝白牦牛)的遗传变异和关系进 行综合分析, 表明 $94.4 \%$ 的遗传变异存在品种内, $5.6 \%$ 的遗传变异存在于品种间, 各牦牛品种(群体) 具有丰富的遗传多样性。田应华等 ${ }^{[32]}$ 基于 15 个SSR 标记对中甸牦牛进行分析, 表明其群体遗传变异丰 富。此外, Qi 等 ${ }^{[33]}$ 使用SSR标记结合mtDNA序列对 中国、不丹、蒙古、俄罗斯等 8 个国家的 29 个群体 共 1076 个家牦牛个体中普通牛的基因渐渗现象进 行了分析, 结果提示在青藏高原、蒙古和俄罗斯牦 牛产区中普通牛基因渐渗水平较高, 而在喜马拉雅 和帕米尔地区渐渗水平较低; 但渐渗水平高低与海 拔高度无显著相关。同时, 冯冬梅等 ${ }^{[34]}$ 分析了牦牛、 普通牛和瘤牛在ILSTS013、ILSTS050 和SPS115 3 个微卫星座位上存在的特异性等位基因, 表明 3 个 牛种在这 3 个基因座上等位基因的DNA序列存在种 间特异性差异,依据该特点可将 3 个牛种进行区分。 可以看出, SSR标记研究结果揭示中国所有家牦牛 $\left(\mathrm{H}_{\mathrm{O}}: 0.550 \sim 0.730 ; \mathrm{H}_{\mathrm{E}}: 0.592 \sim 0.710\right)$ 、野牦牛 $\left(\mathrm{H}_{\mathrm{O}}\right.$ : $\left.0.670 ; \mathrm{H}_{\mathrm{E}}: 0.470\right)$ 、不丹牦牛 $\left(\mathrm{H}_{\mathrm{O}}: 0.598 \sim 0.621 ; \mathrm{H}_{\mathrm{E}}\right.$ : $0.644 \sim 0.680)$ 、蒙古牦牛 $\left(\mathrm{H}_{\mathrm{O}}: 0.625 \sim 0.705 ; \mathrm{H}_{\mathrm{E}}\right.$ : $0.618 \sim 0.690)$ 和俄罗斯牦牛 $\left(\mathrm{H}_{\mathrm{O}}: 0.641 ; \mathrm{H}_{\mathrm{E}}: 0.659\right)$ 品种(群体)均具有丰富的遗传多样性, 但区域间遗 传多样性指数大小相差不大; 更多的遗传变异存在 于各牦牛品种(群体)内, 品种(群体)间存在较显著的 遗传分化; 支持将中国牦牛划分为青藏高原型和横 断高山型两个类型的观点。同时, 也提示相比青藏 高原、蒙古和俄罗斯牦牛群体, 喜马拉雅和帕米尔 地区的牦牛群体具有较低的普通牛基因渐渗水平。

综上所述，基于上述各种分子标记对牦牛分子 
遗传多样性及其他方面的研究现状, 其概况可归纳 为以下几点: (1)研究者探究了普通牛各种分子标记 在牦牛研究中的可行性, 当前已在牦牛研究中使用 的多态性 RAPD、AFLP、SRAP、SSR 标记分别达 31、11、4、48 个; (2)基于上述标记, 学者们已对中 国、不丹、印度、蒙古和俄罗斯的多个牦牛品种(群 体)进行了较为深入的群体遗传分析; (3)探索了可用 于识别和鉴定牦牛、普通牛及犏牛不同牛种的 3 个 特定 SSR 标记, 即 ILSTSO 13、ILSTSO50 和 SPS115; (4) 结合测序技术, 利用苂光标记的 SSR 标记发展了二 重、三重和四重 PCR 方法, 将其运用于牦牛的群体 遗传学和亲子鉴定分析; (5)基于上述标记探究了牦 牛品种(群体)的分类、类型划分、分化状况、起源地、 分类学地位和系统发育关系等问题; (6)除对家牦牛 进行了较为详细的遗传多样性分析, 已初步基于 SSR 标记对少量野牦牛个体进行了遗传多样性研究; (7)基于双重标记(即 SSR 标记和 mtDNA 序列)对涵盖 几乎整个牦牛产区的多个牦牛品种(群体)中普通牛 的基因渐渗现象进行系统分析。整体来看，基于 SSR 标记对牦牛分子遗传多样性的研究相对较多。鉴于 SSR 标记结果的准确性、重复性和可靠性较其他分 子标记高, 故以上有关牦牛 SSR 标记的研究结果今 后理应得到充分重视, 其研究结果可为来自其他角 度对牦牛群体遗传学研究的结果提供信息比较与 参考。

\subsubsection{Y 染色体分子标记}

动物Y染色体具有遵循父系遗传、单倍型完整、 突变率低、不易受重组和回复突变影响的特点, 是 研究动物父系遗传多样性、父系起源及迁徙路线的 理想标记。近年来, 基于Y染色体SSR标记, 学者们 对牦牛的父系遗传多样性也进行了初步研究。 Edwards 等 ${ }^{[35]}$ 发现INRA126 标记在普通牛中为Y染色 体特异SSR标记, 而在牦牛中为非特异Y染色体 SSR 标记。Han等 ${ }^{[36]}$ 利用 3 个普通牛Y染色体特异SSR标 记检测了 1 个不丹牦牛群体和 4 个中国牦牛群体(即 甘南、大通、天祝白牦牛和天祝黑牦牛), 发现标记 INRA126 在公、母牦牛中均有PCR扩增产物; BM861 和INRA189 不仅是存在于普通牛和牦牛中的Y染色 体特异SSR标记, 且各标记拥有检测普通牛、瘤牛和 牦牛的不同等位基因; INRA189 在牦牛群体中为拥
有 3 个复等位基因的多态标记。而Qi等 ${ }^{[37]}$ 则进一步 发现BM861 标记表现为多态, BYM-1 在牦牛中也表 现为多态性的Y染色体特异SSR标记。然而, Nguyen 等 ${ }^{[26]}$ 研究表明, 普通牛Y染色体特异INRA126、 $B M 861$ 标记在公、母牦牛中均有PCR扩增产物, 而 INRA124 和INRA189 两个标记只在公牦牛中有PCR 扩增产物。

可以看出，基于 Y 染色体 SSR 标记对牦牛群体 遗传多样性的研究相对较少, 但可喜的是研究者已 探明了存在于牦牛 Y 染色体上的 3 个特异 SSR 标记 (即 INRA124、INRA189 和 BYM-1)，这为今后评估牦 牛父系遗传多样性奠定了基础。但就目前的研究现 状来看, 只局限于对部分中国、不丹和瑞士的牦牛 群体 Y 染色体特异 SSR 标记遗传多样性分析, 缺乏 对整个牦牛产区群体的系统综合研究。

\section{2 核基因组侯选基因}

近年来，人们已对与牦牛生长发育 (如 $G H$ 、 GHR、GHRH、IGF、LEPR、PIT-1、OB、MC4R、

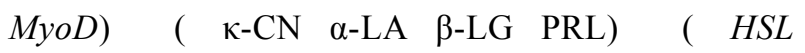
$F A B P 、 L P L 、 M S T N 、 M y f-5 、 C A P N 、 C A S T 、 P R K A \mathrm{G} 3 、$ $F A S) 、$ 毛(如 $M C 1 R) 、$ 繁殖(如 $F S H R 、 G D F-9 、 Z F X / Z F Y$ 、 ZP-3、SRY、SYCP3、TRO、INHA、 hNRNPK、BOULE、 $D A Z L)$ 、抗病免疫 (如 $L F 、 T N F-a 、 T G F-\beta 2$ 、 $H E P C I D I N 、 M H C) 、$ 适应性(如HSP72、EPO、ATP、 $H I F-1 \alpha 、 G L O B I N 、 M B 、 E P A S 1 、 L D H 、 V E G F)$ 等 相关基因进行了分子结构特征、多态性和表达分析。 通过采用PCR-RFLP、PCR-SSCP标记和直接测序的 方法, 分析上述侯选基因序列在不同牦牛品种(群体) 内及品种(群体)间个体的差异, 发现单核苷酸多态 (SNP, Single nucleotide polymorphism)位点, 是揭示 特牛种内分子遗传多样性的另一研究途径。在分析 侯选基因在牦牛种内分子遗传多样性方面, 近年来 有部分侯选基因的研究相对比较深入。如对牦牛的 生长激素 $(G H)$ 基因, 自姬秋梅等 ${ }^{[38]}$ 最早克隆这一基

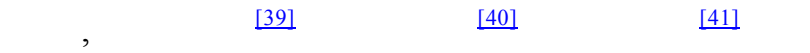
永红等 ${ }^{[42]}$ 和白晶晶等 ${ }^{[43]}$ 先后对麦洼、九龙、巴州、 西藏、青海高原、天祝白牦牛 $G H$ 基因的多态性进行 分析, 结果在麦洼牦牛 $G H$ 基因 $3^{\prime}$-端侧翼区发现一 $M s p$ I / HpaII酶切多态位点; 在巴州牦牛 $G H$ 基因序 列中发现两个变异位点; 发现青海高原、天祝白牦 
牛 $G H$ 基因第 1 、第 2 和第 4 外显子无多态性, 第 3 内含子发生一 $\mathrm{T}>\mathrm{C}$ 转换; 天祝白牦牛第 5 外显子发 生一 $\mathrm{G}>\mathrm{T}$ 颠换。在对牦牛黑素皮质素受体-4(MC4R) 基因的多态性分析中，自宋海峰等 ${ }^{[44]}$ 对该基因进行克 隆和测序后, $\mathrm{Cai}$ 等 $^{[45]}$ 和 $\mathrm{Ma}$ 等 ${ }^{[46]}$ 先后对家、野牦牛 $M C 4 R$ 基因编码区进行多态性检测，发现了 $\mathrm{C}>\mathrm{T}$ 和 $\mathrm{C}>\mathrm{A}$ 两个SNP位点, 确定了 3 种单倍型。在肉质侯选 基因的研究中，马志杰等 ${ }^{[47]}$ 对九龙、麦洼和巴州牦 牛的激素敏感脂肪酶(HSL)基因部分外显子I进行分 析，表明牦牛在 $H S L$ 基因第 1 外显子内具有 $-\mathrm{G}>\mathrm{A}$ $\mathrm{SNP}$ 决定的 $S m a I$ 酶切多态性, 导致甘氨酸 $(\mathrm{G})$ 变为精 氨酸 (R); 在该酶切位点, 3 个牦牛群体均处于 Hardy-Weinberg平衡状态; 九龙与麦洼、巴州牦牛在 等位基因频率上差异显著, 而麦洼与巴州牦牛之间 差异不显著。在与免疫和抗病性相关侯选基因研究 中, $A n$ 等 ${ }^{[48]}$ 对甘南和天祝白牦牛Bogr-DRA 基因第 2 外显子进行多态性分析, 共发现 4 个核苷酸变异位 点, 确定了 5 种单倍型, 其中甘南牦牛拥有 3 种单倍 型, 而天祝白牦牛可能因瓶颈效应的影响只拥有 2 种单倍型。此外, 人们也初步探究了某些基因多态性 与生产性状的关联。如Chen等 ${ }^{[49]}$ 分析了九龙、麦洼和 天祝白牦牛 $M C 1 R$ 基因变异与毛色的关系, 结果在 编码区检测到 5 个核苷酸变异, 确定了EY1、EY2 和EY3 3 种单倍型, 但这 3 种单倍型与黑白毛色不相 关。随后，唐慗挺等 ${ }^{[50]}$ 、高旭东等 ${ }^{[51]}$ 和 Xi等 ${ }^{[52]}$ 先后 对麦洼、斯布、九龙、西藏、甘南、大通、青海高 原、天祝白牦牛以及犏牛 $M C 1 R$ 基因内的变异位点进 行检测, 共发现 13 个SNP位点, 揭示各牦牛品种(群 体)间以及与犏牛在该基因序列上存在一定差异。

当然, 目前对牦牛侯选基因的多态性研究较多, 但大多不够系统，故在此不逐一阐述。但从目前的 整体研究来看, 大多集中于与牦牛生长发育、乳、 肉、毛、繁殖、抗病、适应性等密切相关的基因。 研究内容除进行了基因的分子特征、表达和多态性 研究外, 当前初步开展了部分侯选基因多态性与生 产性能的关联分析, 这是一个可喜的开始。但值得 注意的是, 在牦牛产区很难获得可靠的生产性能数 据资料，因此，在开展基因多态性与生产性能的关 联分析时需要谨慎。此外, 由于大多候选基因相对 比较保守, 就目前关于侯选基因在牦牛种内的遗传 多样性研究方面, 存在的一个突出的问题: 即除对
个别基因达到了对其全序列的多态性系统检测外, 而大多数研究只停留于对部分基因片段在少数牦牛 品种(群体)中的检测。因此，今后有必要针对侯选基 因的全序列，探讨等位基因或单倍型在不同牦牛品 种(群体)的频率分布, 进而揭示其遗传多样性。此外, 对与牦牛经济性状相关侯选基因的定位和表达调控 等亦是今后研究的重点。

\section{2 线粒体基因组(mtDNA)的遗传多样性}

动物线粒体基因组 (mtDNA) 具有进化速率快、 多态性丰富、无重组、遵循母系遗传等特点, 被认 为是当前探究动物起源进化、分类、群体遗传多样 性等研究的一种很好的分子标记。近年来，从 mtDNA序列探究牦牛的群体遗传多样性成为科研人 员的主要研究策略之一。Zhao等 ${ }^{[53]}$ 利用 mtDNA RFLP方法分析比较了九龙牦牛、麦洼牦牛、犏牛和 普通牛之间的关系, 结果提示 $B a m \mathrm{H}$ । 位点在九龙 和麦洼牦牛间存在差异, 犏牛mtDNA 限制性图谱 与麦洼牦牛一致。与此同时, 他还对牦牛和普通牛 的mtDNA ATPase 6 和ATPase 8 进行RFLP和SSCP分 析 ${ }^{[54]}$, 表明普通牛在 6 个限制性内切酶RFLP分析中 呈现多态, 而牦牛为单态; SSCP结果提示在牦牛和 普通牛种间和种内均具有高度多态。涂正超等 ${ }^{[55]}$ 用 20 种限制性内切酶分析了中国九龙、麦洼、青海环湖、 中甸和天祝白牦牛 5 个群体 90 个个体的 $\mathrm{mtDNA}$ RFLP，并利用双酶切制定出其物理图谱，表明 6 种 酶切类型具有多态性, 共发现 5 种 mtDNA单倍型, 提示上述牦牛群体mtDNA多态性较贫乏，群体间的 分化程度较低, 估计牦牛和普通牛、瘤牛的分化时 间大约分别在 1.1 2.2 百万年和 1.01 1.02 百万年之 间。Bailey等 [56]测定了中国、不丹、尼泊尔和蒙古 的部分牦牛群体mtDNA D-loop区序列, 结果表明牦 牛可能存在双驯化模式, 这些牦牛群体具有较高的 遗传多样性水平，但缺乏明显的系统地理分布特 征。Han等 ${ }^{[361}$ 检测了 1 个不丹特牛群体和 4 个中国 牦牛群体中普通牛的渐渗状况, 发现 239 头牦牛个 体中只有 3 头母牦牛携带普通牛 mtDNA, 说明普通 牛的渐渗水平较低。赖松家等 $[57,58]$ 先后测定 了中国 5 个牦牛群体和少量犏牛个体的 mtDNA D-loop区全序列和 $C y t b$ 部分序列，对其遗传多样性 和起源驯化进行综合分析, 结果表明 5 个牦牛群体 
具有丰富的遗传多样性; 推测中国牦牛起源于同一 祖先, 可能有两个主要的驯化地点或来自具有两个 分化支系的杂合基因库。李齐发等 ${ }^{[59,60]}$ 基于mtDNA D-loop区和 $C y t b$ 基因对牦牛的分类学地位进行研究, 结果表明家、野牦牛首先与美洲野牛聚为一类, 牦 牛与美洲野牛属间的关系较之牛属其他物种更近, 支持将家、野牦牛划分为牛亚科中一个独立属即牦 牛属的观点。郭松长等 ${ }^{[61 ~ 63]}$ 测定了中国 10 个家牦牛 品种和 1 个野牦牛群体mtDNA D-loop区部分序列 (636 637 bp), 探讨了其起源驯化、遗传多样性、分 化、属级分类学地位, 表明家、野牦牛均具有丰富 的遗传多样性; 中国的家牦牛存在两个聚类簇, 品 种间具有显著的遗传分化; 不支持将牦牛独立为牦 牛属, 认为牛属与野牛属在分类上也应合并为一个 属; 牦牛与野牛属的分化发生在欧亚大陆, 冰期结 束后, 欧亚大陆其它地区温度升高, 牦牛只能局限 分布在较为寒冷的青藏高原。与此同时, Qi等 ${ }^{[64]}$ 对分 布于中国、不丹、尼泊尔、印度、巴基斯坦、哈萨 克斯坦、蒙古和俄罗斯的 29 个牦牛群体共 428 个个 体mtDNA D-loop区和Cyt $b$ 基因全序列进行了综合 分析, 系统聚类显示有 3 个大的牦牛支系, 推测各 支系间分化时间在 63000 136100 BP之间; 3 个支系 中有一个支系发生群体扩张事件, 大约发生在 5000 $\mathrm{BP}$, 与牦牛驯化的考古学证据一致; 在四个组群中, 青藏高原产区的牦牛群具有最高的单倍型多样性, 蒙古和俄罗斯产区以及喜马拉雅山东部的牦牛群次 之, 而喜马拉雅山西部牦牛群最低; 现今的牦牛群 体约在 5000 BP经历了一个单个的驯化事件, 驯化 前至少有 3 个支系的野牦牛群生活在不同的避难所, 青藏高原东部可能是牦牛的驯化点; 家牦牛可能有 两条迁徙路线：一是自青藏高原东部西经喜马拉雅 和昆仑山到“帕米尔结”地区，二是自青藏高原东部 直接经蒙古的南戈壁和戈壁阿尔泰山到过蒙古和俄 罗斯。马志杰等 ${ }^{[65,66]}$ 对野牦牛的mt DNA D-loop区部 分序列进行分析, 结果表明野牦牛群体具有丰富的 遗传多样性, 聚类显示有两个高度分化的遗传分 支。杨万远等 ${ }^{[67]}$ 也测定了野牦牛 Cyt $b$ 基因全序列, 构建了野牦牛等牛亚科种间系统进化树, 结果表明 野牦牛 $C y t b$ 基因序列间共有 13 个SNP多态位点, 支 持将牦牛划分为牛亚科中一个独立属的观点。而常 国斌等 ${ }^{[68]}$ 对巴州牦牛 $C y t b$ 基因部分序列进行分析,
共发现 7 个变异位点, 定义了 4 种单倍型; 巴州牦牛 可能具有 2 种母系起源。此外，Wang等 60970 综合分 析了中国 405 头家牦牛以及 47 头野牦牛的 $\mathrm{mtDNA}$ D-loop区、48 头家牦牛和 21 头野牦牛的mtDNA全 序列以及 51 头家牦牛和 21 头野牦牛的 $\mathrm{mtDNA}$ 蛋白 编码序列, 结果表明野牦牛的单倍型多样性以及核 普酸多样性都高于家牦牛; 共检测到 3 个高度分化 的遗传分支, 其中两大主要分支在家、野牦牛中都 有分布, 第三个分支包含的个体数较少, 只分布在 野牦牛居群之中; 家牦牛中没有明显的谱系地理结 构; 牦牛种内三大分支的分化时间估计在 42 万年 ５8 万年之间，与青藏高原第四纪冰期事件发生的 时间相吻合; 线粒体蛋白编码序列分析发现家牦牛 分支的非同义突变率显著地高于野牦牛分支, 一定 程度上提示驯化以及驯化过程中的瓶颈效应导致了 家牦牛线粒体所受选择压力减小; 同时, 研究还对 17 头野牦牛和 32 头北美野牛的线粒体基因组蛋白 编码序列进行了分析, 发现在牦牛线粒体蛋白跨膜 螺旋区域中存在苏氨酸残基的增加，推测该苏氨酸 残基的增加可能与牦牛对高海拔环境的适应有关。 赵上娟等 ${ }^{[71]}$ 对 11 个西藏牦牛类群 111 个个体的 mtDNA COIII的全序列进行测定分析，表明西藏牦 牛具有较丰富的mtDNA遗传多样性, 11 个牦牛类群 可分为 3 大类, 研究支持将牦牛独立为牦牛属的观 点。张成福等 ${ }^{[72]}$ 测定和分析了西藏 11 个牦牛类群 114 个个体的mtDNA D-loop区全序列，结果表明西 藏牦牛具有丰富的单倍型类型; 聚类分析和 AMOVA 分析显示西藏牦牛可分为两大类, 康布牦 牛、嘉黎牦牛为一类, 其余的牦牛类群为另一类。 马云等 ${ }^{[73]}$ 对青海高原牦牛和环湖牦牛 2 个牦牛品种 的mtDNA D-loop区全序列进行分析，结果提示青海 高原牦牛与野牦牛的亲缘关系较近, 环湖牦牛与家 牦牛的亲缘关系较近。

可以看出，基于 mtDNA 序列学者们就以下内 容进行了系统分析 : (1) 利用 RFLP、SSCP 和测序技 术，基于 mtDNA 不同的基因片段及全序列，从不同 的角度对几乎整个牦牛产区的多个牦牛品种(群体) 进行了较为深入的群体遗传学分析, 特别是对中国 牦牛品种(群体)的研究报道较多且比较系统; (2)除对 牦牛的遗传多样性分析外, 还探究了牦牛的分类、 分化状况、起源驯化模式、分类学地位、系统发育 
关系、群体历史发展动态、谱系地理结构等问题; (3) 分析了家、野牦牛与普通牛、犏牛在遗传多样性上 的差异，探究了可用于开展亲子鉴定的分子标记; (4)对牦牛品种(群体)中普通牛的基因渐渗现象进行 了深入探究。整体来看, 基于 mtDNA 序列的研究得 出了以下几点富有结论性的成果 : (1)家、野牦牛均 具有丰富的遗传多样性。相比而言, 野牦牛群体 [ D-loop 区(Hd：0.9600 1.0000; $\pi: 0.0221 \sim 0.0244$ ); mtDNA 全序列(Hd : 0.9910; $\pi: 0.0037)]$ 比家牦牛群 体[D-loop 区(Hd :0.8800 0.9251; $\pi: 0.0035 \sim 0.0156$ ); mtDNA 全序列(Hd : 0.9920; $\pi: 0.0029)]$ 具有较高的 遗传多样性水平; 家牦牛多样性以青藏高原产区的 群体水平最高, 蒙古和俄罗斯产区及喜马拉雅山东 部群体次之, 喜马拉雅山西部群体最低; (2)青藏高 原东部可能是牦牛的驯化点, 不支持青藏高原北部 作为驯化点的结论, 但具体的驯化点在中国的青海 省还是西藏自治区, 或是兼而有之, 目前仍无定论; 推测牦牛驯化发生在约 $5000 \mathrm{BP}$, 经历了一个单个 的驯化事件; (3)家、野牦牛均有两个或 3 个高度分化 的遗传分支，且各分支分化时间发生在其驯化事件 以前; (4)更多的支持将牦牛当作一个独立属的观点; (5)家牦牛品种(群体)间具有不同程度的遗传分化,但 不显著。更多的遗传变异存在于各牦牛品种(群体) 内, 品种(群体)间无明显的谱系地理结构,其遗传距 离与地理分布无明显相关; (6)家牦牛的迁徙路线有 两种可能，一是自青藏高原东部西经喜马拉雅和昆 仑山到“帕米尔结”地区，二是自青藏高原东部直接 经蒙古的南戈壁和戈壁阿尔泰山到蒙古和俄罗斯等 地; (7)家牦牛 mtDNA 非同义突变率显著地高于野牦 牛, 牦牛线粒体蛋白跨膜螺旋区域中存在苏氨酸残 基的增加; 8)牦牛群体中普通牛的基因渐渗程度总 体较低; (9)支持将中国家牦牛划分为两大类型即横 断高山型和青藏高原型的观点。

\section{3 结 语}

综观分子水平上的牦牛遗传多样性研究成果, 可以看出家、野牦牛均具有丰富的遗传多样性; 家 牦牛品种(群体)间具有不同程度的遗传分化,无明显 的谱系地理结构,更多的遗传变异存在于各品种(群 体)内。这些结论为整体了解世界牦牛品种(群体)遗 传资源状况以及开展其合理的保护和开发利用奠定
了理论基础。尽管如此, 在分析近年来有关牦牛分 子遗传多样性研究的基础资料上，结合笔者们对该 领域相关问题的研究与思考, 认为仍存在以下若干 问题和不足，有待今后继续思考与探究：(1)尽管利 用常染色体分子标记对多个牦牛品种(群体)遗传多 样性进行了分析, 但受所使用的分子标记类型、实 验条件以及所研究的牦牛品种(群体)不同等因素影 响, 大多数研究结果缺乏可比性。因此, 今后研究中, 建议尽可能地使用不同标记的结合, 特别是SSR标 记与mtDNA序列的结合策略, 并收集世界牦牛分布 区尽可能多的样本来开展该研究领域的工作。(2)目 前基于SSR标记研究者多采用五重以下的PCR技术, 这在一定程度上耗费人力、物力和时间。随着新一 代测序技术的发展，发展五重以上的PCR技术并将 其用之于牦牛遗传多样性研究是可行的。由此, 发 展五重以上的多重PCR技术将是今后开展该领域研 究省钱、省力和省时的策略之一。(3)尽管人们已发 现 3 个牦牛Y染色体特异SSR标记，但一则被探明的 牦牛Y染色体特异SSR标记较少, 二则缺乏对所有 牦牛品种(群体)Y染色体特异SSR标记的遗传多样性 分析。同时, 从Y染色体上特异基因的SNPs位点这一 角度对牦牛的遗传多样性研究国内外尚未见报道。 由此, 在今后研究中, 一是继续挖掘更多的牦牛Y 染色体特异SSR标记，二是开展基于Y染色体特异 SSR标记以及特定基因的单倍型分析进行综合系统 研究, 将是探究牦牛父系遗传多样性及起源等问题 的研究重点。(4)目前从mtDNA全序列对牦牛群体的 遗传多样性研究还相对较少 ${ }^{[69,70]}$ 。随着测序费用的 降低, 今后通过测定所有牦牛mtDNA全序列, 分析 $m t D N A$ 全序列信息基础上的牦牛遗传多样性将是从 母系遗传角度深入探究该问题的另一目标。(5)相比 家牦牛分子遗传多样性研究, 野牦牛的研究相对较 少 ${ }^{[20,21,61,65 ~ 67,69,70]}$, 且在研究分析中样本量较小缺乏 代表性, 这在一定程度上影响对其遗传多样性的准 确评估。因此, 今后有必要通过采集野牦牛粪便、被 毛等方法加大样本量来开展其多样性的评定。

最近牦牛全基因组序列已测定和公布 ${ }^{[74]}$, 在获 得总长为 $2657 \mathrm{Mb}$ 母牦牛全基因组序列基础上, 发 现牦牛全基因组序列 GC含量分布类似于普通牛基 因组的全序列; 推测牦牛基因组中蛋白编码基因为 22282 个; 共鉴定出 220 万个杂合单核苷酸变异体 
(Single nucleotide variants, SNVs), 其杂合率 $(0.89 \times$ $\left.10^{-3}\right)$ 是普通牛 $\left(0.59 \times 10^{-3}\right)$ 的 1.5 倍; 确定牦牛、普通 牛、人和狗共有的同源基因家族 13810 个，其中 100 个(包括 170 个基因)为牦牛所特有; 推测牦牛和普通 牛约在 490 万年前就已分化; 在牦牛中共鉴定出 85 个正向选择基因(Positive selected genes, PSGs)均与 缺氧应激和能量代谢有关, 包括两个重要的调控子 (Adam17 和 $\operatorname{Arg} 2)$ 和一个靶基因 (Mmp3)与缺氧功能 有关，而 5 个重要基因(Camk2b、Gcnt3、Hsd17b12、 $W h s c 1$ 和 $G l u l$ )在牦牛营养代谢通路中显示出正向选 择信号。今后可在牦牛全基因组序列信息基础上, 挖掘一大批分子标记, 并将其用之于牦牛群体遗传 多样性评估。同时，在上述获得的杂合单核苷酸变 异位点基础上, 进一步探究世界不同牦牛品种(群体) 在全基因组水平上的单核苷酸多样性(SNP)。此外, 未来的研究重心应转移到利用新一代测序技术和基 因芯片以及全基因组关联分析等，发现新的功能基 因，并通过高通量的测序和高通量分子标记的使用 来挖掘全基因组水平上特牛遗传多样性的状况，进 行为开展牦牛分子育种、遗传资源的保护和利用等 实践提供科学依据和理论指导。

\section{参考文献(References):}

[1] Prezewalski NM. From Zaisan Lake through the Kham region of Tibet and the head of the Yellow River. 2 nd ed. Moscow, 1948. DOI

[2] Wiener G, Han JL, Long RJ. The Yak. 2nd ed. Bangkok, Thailand: Regional Office for Asia and the Pacific of the Food and Agriculture Organization of the United Nations, 2003. DOI

[3] Han JL. Yak genetic resources in China. In: Proceedings of the regional workshop on conservation and management of yak genetic diversity. ICIMOD, Kathmandu, Nepal, 1996. DOI

[4] Steane DE. Conservation and use of animal genetic resources in Asia-its relevance to yak. In: Proceedings of the Second International Congress on Yak. Xining, Qinghai: Qinghai People's Publishing House, 1997: 43-49. DOI

[5] Wiener G. Yak breeds and the utilization of genetic diversity. In: Proceedings of the Second International Congress on Yak. Xining, Qinghai: Qinghai Peopli's Publishing House, 1997: 50-53. DOI

[6] Zhong JC. The genetic diversity of Chinese yak and its significance. In: Proceedings of the Second International
Congress on Yak. Xining, Qinghai: Qinghai People's Publishing House, 1997: 65-69. DOI

[7] 欧江涛, 钟金城, 白文林, 赵素君. 中国牦牛的遗传多 样性. 黄牛杂志, 2002, 28(4): 42-46. DOI

[8] Groeneveld LF, Lenstra JA, Eding H, Toro MA, Scherf B, Pilling D, Negrini R, Finlay EK, Han JL, Groeneveld E, Weigend S. Genetic diversity in farm animals-a review. Anim Genet, 2010, 41(s1): 6-31. DOI

[9] 魏亚萍, 张周平. 牦牛、黑白花牛及其远缘杂种RAPD 标记的初步研究. 黄牛杂志, 1999, 25(5): 16-18. DOI

[10] 伍红, 饶开晴, 钟金城, 陈智华, 欧江涛. 麦洼牦牛的 RAPD分析．四川畜牧兽医, 2002, 29(9): 19-20. DOI

[11] 江明锋, 姜权. AFLP分子标记在牦牛遗传分析中的初探. 四川畜牧兽医, 2003, 30(11): 20-21. DOI

[12] 钟金城, 陈智华, 赵素君, 肖玉萍. 牦牛生态类型的分 类. 生态学报, 2006, 26(7): 2068-2072. DOI

[13] 肖玉萍, 钟金城, 金双. 4 个牦牛品种的RAPD遗传多样 性研究. 中国牛业科学, 2007, 33(6): 5-10. DOI

[14] 肖玉萍, 钟金城, 魏云霞, 马志杰, 王华东. 牦牛品种 的AFLP分析及其遗传多样性研究. 中国草食动物, 2008, 28(2): 12-15. DOI

[15] 钟金城, 柴志欣, 姬秋梅, 赵尚娟, 徐利娟, 唐懿挺, 张 成福, 信金伟. 西藏牦牛的遗传多样性及其系统进化研 究. 西南民族大学学报(自然科学版), 2011，37(3): 368378. DOI

[16] 柴志欣，赵上娟，姬秋梅，张成福，信金伟，钟金城. 西 藏牦牛的RAPD遗传多样性及其分类研究. 畜牧兽医学 报, 2011, 42(10): 1380-1386. DOI

[17] 赵上娟，钟金城，柴志欣，张成福，信金伟，陈智华. 西 藏牦牛SRAP遗传多样性及分类进化研究. 生物技术通 报, 2012, (6): 77-82. DOI

[18] Ramesha KP, Kumar KVP, Chandrashekar K, Das S, Saravanan BC, Pourouchottamane R, Kataktalware M. Genetic variability of Indian yaks using random amplified polymorphic DNA markers. Afr J Biotechnol, 2011, 10(43): 8558-8561. DOI

[19] Ritz LR, Glowatzki-Mullis ML, MacHugh DE, Gaillard C. Phylogenetic analysis of the tribe Bovini using microsatellites. Anim Genet, 2000, 31(3): 178-185. DOI

[20] 王敏强. 大通牦牛部分种质特性研究[学位论文]. 西北 农林科技大学, 2000. DOI

[21] 胡江. 野牦牛、家牦牛及其杂交种的生物学特性和遗传 多样性研究[学位论文]. 甘肃农业大学, 2001. DOI

[22] Dorji T, Goddard M, Perkins J, Robinson N, Roder W. Genetic diversity in Bhutanese yak (Bos grunniens) Populations using microsatellite markers. In: Proceedings of the third International Congress on Yak. Lhasa, Tibet, China, 2000: 197-201. DOI

[23] Wang MQ, Weigend S, Barre-Dirie A, Carnwath JW, Lu ZL, Niemann H. Analysis of two Chinese yak (Bos grunniens) populations using bovine microsatellite primers. $J$ 
Anim Breed Genet, 2003, 120(4): 237-244. DOI

[24] Qi XB. Genetic diversity, differentiation, and relationship of domestic yak populations: a microsatellite and mitochondrial DNA study. PhD dissertation, Lanzhou University, Gansu. 2004. DOI

[25] Li QF, Zhao ZB, Li YH, Li N, Wu CX, Xie Z. Construction of microsatellite-enriched library of yak and phylogenetic study of six Chinese yak populations using yak-specific microsatellites. In: Proceedings of the fourth International Congress on Yak. Chengdu, Sichuan, China, 2004: 109-111. DOI

[26] Nguyen TT, Genini S, Ménétrey F, Malek M, Vögeli P, Goe MR, Stranzinger G. Application of bovine microsatellite markers for genetic diversity analysis of Swiss yak (Poephagus grunniens). Anim Genet, 2005, 36(6): 484-489. DOI

[27] Qi XB, Han JL, Lkhagva B, Chekarova I, Badamdorj D, Rege JE, Hanotte O. Genetic diversity and differentiation of Mongolian and Russian yak populations. J Anim Breed Genet, 2005, 122(2): 117-126. DOI

[28] 钟金城，赵素君，陈智华，马志杰. 牦牛品种的遗传多样 性及其分类研究. 中国农业科学, 2006, 39(2): 389-397. DOI

[29] 毛永江, 常洪, 杨章平, 张柳, 宋光明, 王小龙. 青海高 原牦牛遗传多样性研究. 家畜生态学报, 2008, 29(1): 25-31. DOI

[30] Zhang GX, Chen WS, Xue M, Wang ZG, Chang H, Han X, Liao XJ, Wang DL. Analysis of genetic diversity and population structure of Chinese yak breeds (Bos grunniens) using microsatellite markers. J Genet Genomics, 2008, 35(4): 233-238. DOI

[31] 廖信军, 常洪, 张桂香, 王冬蕾, 宋卫涛, 韩旭, 张自富. 中国 5 个地方牦牛品种遗传多样性的微卫星分析. 生物 多样性, 2008, 16(2): 156-165. DOI

[32] 田应华, 钱林东, 凌军, 刘丽仙, 霍金龙, 祁宏, 苗永旺 云南中甸牦牛的遗传多样性研究. 西南农业学报, 2009 , 22(3): 794-797. DOI

[33] Qi XB, Han JL, Wang G, Rege JEO, Hanotte O. Assessment of cattle genetic introgression into domestic yak populations using mitochondrial and microsatellite DNA markers. Anim Genet, 2010, 41(3): 242-252. DOI

[34] 冯冬梅, 赵会静, 罗玉柱, 韩建林. 牦牛和黄牛三个微 卫星基因座种间特异性等位基因分析. 甘肃农业大学 学报, 2010, 45(4): 22-27. DOI

[35] Edwards CJ, Gaillard C, Bradley DG, MacHugh DE. Y-specific microsatellite polymorphisms in a range of bovid species. Anim Genet, 2000, 31(2): 127-130. DOI

[36] Han JL, Ochieng JW, Rege JEO, Hanotte O. Low level of cattle introgression in yak populations from Bhutan and China: Evidences from Y-specific microsatellites and mitochondrial DNA markers. In: Proceedings of the third International Congress on Yak. Lhasa, Tibet, China, 2000:
190-196. DOI

[37] Qi XB, Han JL, Rege JEO, Hanotte O. Y-Chromosome specific microsatellite polymorphisms in Chinese yak. In: Proceedings of $7^{\text {th }}$ World Congress on Genetics Applied to Livestock Production. Montpellier, France, 2002: 509-512. DOI

[38] Ji QM. Construction of yak genomic library and dissociated $G H$ gene. In: Proceedings of the First International Congress on Yak. Lanzhou: Printing House of Gansu Economic Management Cadre Institute, 1994: 102-103. DOI

[39] 陈桂芳, 李齐发, 强巴央宗, 土登, 谢庄。西藏牦牛和 黑白花奶牛生长激素基因AluI多态性的比较研究. 畜牧 与兽医, 2002, 34(11): 15-16. DOI

[40] 欧江涛，钟金城，赵益新，陈智华. 牦牛生长激素基因 的测序和多态性研究. 黄牛杂志, 2003, 29(2): 9-12. DOI

[41] 毛晓玲, 杨易, 刘文静, 金素钰, 郑玉才. 牦牛垂体特 异转录因子-1、生长激素和 $\alpha$-乳清蛋白基因多态性的分 析. 四川草原, 2004, (5): 23-25. DOI

[42] 李永红, 常洪, 耿荣庆, 常国斌, 宋光明, 常春芳, 冀德 君. 以 $G H$ 基因多态性探讨中国南北黄牛及牦牛系统. 畜牧兽医学报, 2008, 39(9): 1165-1170. DOI

[43] 白晶晶, 胡江, 成述儒, 王继卿, 罗玉柱. 牦牛 $G H$ 基因 多态性的PCR-SSCP分析. 甘肃农业大学学报, 2010, 45(1): 1-5. DOI

[44] 宋海峰，魏雅萍，马志杰，钟金城，罗晓林，陈雪梅. 牦 牛黑素皮质素受体-4基因的克隆与序列分析. 青海大学 学报(自然科学版), 2009, 27(3): 43-50. DOI

[45] Cai X, Mipam TD, Zhang HR, Yue BS. Abundant variations of $M C 4 R$ gene revealed by phylogenies of Yak (Bos grunniens) and other mammals. Mol Biol Rep, 2011, 38(4): 2733-2738. DOI

[46] Ma ZJ, Song HF, Zhong JC, David S, Wei YP, Sun YG. Molecular characterisation of the wild yak (Bos grunniens mutus) melanocortin receptor-4 (MC4R) gene and a comparative analysis with that of other Bovinae species. $J$ Appl Anim Res, 2012, 40(2): 81-85. DOI

[47] 马志杰, 钟金城, 字向东, 陈智华, 徐惊涛, 金双, 常怀 普, 宋海峰, 刘建否. 三个牦牛群体激素敏感脂肪酶 (HSL) 基因外显子 I 的多态性. 中国农业科学，2009， 42(1): 370-376. DOI

[48] An TW, Zhang H, He JW, Feng HY, Luo YZ, Han JL. Polymorphic but highly conserved Bogr-DRA gene in yak (Bos grunniens). Anim Genet, 2012, 43(2): 237-238. DOI

[49] Chen SY, Huang Y, Zhu Q, Luca F, Yao YG, Liu YP. Sequence characterization of the MC1R gene in yak (Poephagus grunniens) breeds with different coat colors. $J$ Biomed Biotechnol, 2009, ID 861046, doi:10.1155/2009/ 861046. DOI

[50] 唐懿挺, 钟红梅, 姬秋梅, 张成福, 柴志欣, 赵尚娟, 白 雪，信金伟，钟金城. 牦牛 $M C 1 R$ 基因的克隆测序及其 分析研究. 生物技术通报, 2011, (6): 88-93. DOI 
[51] 高旭东, 余四九, 王明亮, 陈鹏, 郝明超, 刘苼, 王继卿. 四个牦牛品种 $M C 1 R$ 基因部分序列的多态性研究. 生物 技术通报, 2012,(8): 141-145. DOI

[52] Xi DM, Wu M, Fan YY, Huo YQ, Leng J, Gou X, Mao HM, Deng WD. Isolation and characteristics of the melanocortin 1 receptor gene $(M C 1 R)$ in the Chinese yakow (Bos grunniens $\times$ Bos taurus). Gene, 2012, 498(2): 259-263. DOI

[53] Zhao XB, Zhong GH, Cai L. Studies on mitochondrial DNA RFLP of yaks and cattle-yak. In: Proceedings of the first International Congress on Yak. Lanzhou, Gansu, China, 1994: 96-98. DOI

[54] Zhao XB, Wu CX, Li N, Luo XL. PCR-RFLP and SSCP of mt-DNA ATPase6, ATPase 8 in yak and cattle populations. In: Proceedings of the Second International Congress on Yak. Xining, Qinghai, China, 1997: 59-61. DOI

[55] 涂正超, 张亚平, 邱怀. 中国牦牛线粒体DNA多态性及 遗传分化. 遗传学报, 1998, 25(3): 205-212. DOI

[56] Bailey JF, Healy B, Han JL, Sherchand L, Pradhan SL, Tsendsuren T, Foggin JM, Gaillard C, Steane D, Zakharov I, Bradley DG. Genetic variation of mitochondrial DNA within domestic yak populations. In: Proceedings of the third International Congress on Yak. Lhasa, Tibet, China, Nairobi: International Livestock Research Institute (ILRI), 2000: 181-189. DOI

[57] 赖松家, 王玲, 刘益平, 李学伟. 中国部分牦牛品种线 粒体DNA遗传多态性研究. 遗传学报, 2005，32(5): 463-470. DOI

[58] Lai SJ, Chen SY, Liu YP, Yao YG. Mitochondrial DNA sequence diversity and origin of Chinese domestic yak. Anim Genet, 2007, 38(1): 77-80. DOI

[59] 李齐发, 李隐侠, 赵兴波, 刘振山, 张庆波, 宋大伟, 屈 旭光, 李宁, 谢庄. 牦牛线粒体DNA细胞色素b基因序列 测定及其起源、分类地位研究. 畜牧兽医学报, 2006, 37(11): 1118-1123. DOI

[60] 李齐发, 李隐侠, 赵兴波, 潘增祥, 刘振山, 张庆波, 屈 旭光, 宋大伟, 董丽艳, 李宁, 谢庄. 牦牛线粒体DNA D-loop区序列测定及其在牛亚科中分类地位的研究. 畜 牧兽医学报, 2008, 39(1): 1-6. DOI

[61] Guo SC, Savolainen P, Su JP, Zhang Q, Qi DL, Zhou J, Zhong Y, Zhao XQ, Liu JQ. Origin of mitochondrial DNA diversity of domestic yaks. BMC Evol Biol, 2006, 6(1): 73. DOI

[62] 郭松长, 刘建全, 祁得林, 杨洁, 赵新全. 牦牛的分类 学地位及起源研究: mtDNA D-loop序列的分析. 兽类学 报, 2006, 26(4): 325-330. DOI

[63] 郭松长, 祁得林, 陈桂华, 徐世晓, 赵新全. 家牦牛线 粒体DNA(mtDNA) 遗传多样性及其分类. 生态学报, 2008, 28(9): 4287-4294. DOI

[64] Qi XB, Han JL, Blench R, Chekarova I, Rege JE, Hanotte O. Understanding the yak pastoralism in Central Asian
Highlands: genetic evidence for origin, domestication and dispersion of domestic yak. In: Sanchez-Mazas A, Blench R, Ross MD, Peiros I, Lin M (eds.) Past Human Migrations in East Asia: Matching Archaeology, Linguistics and Genetics. London and New York: Routledge, Taylor \& Francis Group. 2008: 427-442. DOI

[65] 马志杰, 钟金城, 韩建林, 徐惊涛, 荬全林, 常怀普. 野 牦牛 (Bos grunniens mutus) mtDNA D-Loop区的遗传多 样性. 生态学报, 2009, 29(9): 4798-4803. DOI

[66] Ma ZJ, Zhong JC, Han JL, Xu JT, Bai WL, Li JY, Cairang DZ, Jin XY, Zhang J. Genetic diversity and demographic history of wild yak (Bos grunniens mutus) inferred from mtDNA D-loop sequences. Afr J Biotechnol, 2010, 9(46): 7805-7810. DOI

[67] 杨万远, 陈雪梅, 钟金城, 陈生梅, 郭承恩, 孟丽, 武甫 德. 野牦牛mtDNA Cytb 基因全序列测定及系统进化关 系. 中国草食动物, 2009, 29(3): 8-13. DOI

[68] 常国斌，常洪，陈国宏，陈蓉，赵荐，卓娅，管永平。基 于 $C y t b$ 基因部分序列分析巴州牦牛遗传多样性及其系 统地位. 中国畜牧杂志, 2010, 46(17): 19-21. DOI

[69] Wang ZF, Shen X, Liu B, Su JP, Takahiro Y, Yu Y, Guo SC, Simon YWHo, Carles V, Masami H, Liu JQ. Phylogeographic analyses of domestic and wild yaks based on mitochondrial DNA: new data and reappraisal. J Biogeogr, 2010, 37(12): 2332-2344. DOI

[70] Wang ZF, Yonezawa T, Liu B, Ma T, Shen X, Su JP, Guo SC, Hasegawa M, Liu JQ. Domestication relaxed selective constraints on the yak mitochondrial genome. Mol Biol Evol, 2011, 28(5): 1553-1556. DOI

[71] 赵上娟, 陈智华, 姬秋梅, 柴志欣, 张成福, 信金伟, 钟 金城. 西藏牦牛mtDNA COIII序列测定及系统进化关系. 中国农业科学, 2011, 44(23): 4902-4910. DOI

[72] 张成福, 徐利娟, 姬秋梅, 信金伟, 钟金城. 西藏牛毛牛 mtDNA D-loop区的遗传多样性及其遗传分化. 生态学 报, 2012, 32(5): 1387-1395. DOI

[73] 马云, 于波, 徐永杰, 吴海港, 梁小娟, 付蕾, 李芬. 中 国部分地方牛种mtDNA D-loop区全序列的遗传多样性 与系统进化分析. 信阳师范学院学报 (自然科学版), 2012, 25(2): 202-217. DOI

[74] Qiu Q, Zhang GJ, Ma T, Qian WB, Wang JY, Ye ZQ, Cao CC, Hu QJ, Jaebum K, Denis ML, Loretta A, Boris C, Ma J, Harris AL, Qian XJ, Lang YS, Zhou R, Wang LZ, Wang K, Xia JQ, Liao SG, Pan SK, Lu X, Hou HL, Wang Y, Zang XT, Yin Y, Ma H, Zhang J, Wang ZF, Zhang YM, Zhang DW, Takahiro Y, Masami H, Zhong Y, Liu WB, Zhang Y, Huang ZY, Zhang SX, Long RJ, Yang HM, Wang J, Johannes AL, David NC, Wu Y, Wang J, Shi P, Wang J, Liu JQ. The yak genome and adaptation to life at high altitude. Nat Genet, 2012, 44(8): 946-949. DOI 